\title{
Threats and outbreaks of cholera in Africa amidst COVID-19 pandemic: a double burden on Africa's health systems
}

\author{
Olivier Uwishema ${ }^{1,2,3^{*}}$ (1) , Melody Okereke ${ }^{4}$, Helen Onyeaka ${ }^{5}$, Mohammad Mehedi Hasan ${ }^{6}$ (D) \\ , Deocles Donatus ${ }^{1,7}$, Zebadiah Martin ${ }^{1,8}$, Lawal Abdulwahab Oluwatomisin ${ }^{1,9}$, Melissa Mhanna ${ }^{1,10}$, \\ Adesipe Olaoluwa Olumide ${ }^{1,11}$, Jeffrey Sun ${ }^{2,12}$ a and Irem Adanur ${ }^{1,3}$
}

\begin{abstract}
Every year, about 4 million cases and 143,000 deaths due to cholera are recorded globally, of which 54\% were from Africa, reported in 2016. The outbreak and spread of cholera have risen exponentially particularly in Africa. Coupled with the recent emergence of the Coronavirus Pandemic (COVID-19) in Africa, the local health systems are facing a double burden of these infectious diseases due to their cumulative impact. In this paper, we evaluate the dual impact of cholera and COVID-19 in Africa and suggest plausible interventions that can be put in place to cushion its impact.

Keywords: Cholera, COVID-19, Africa, Health system, Double burden, Pandemic
\end{abstract}

\section{Background}

While Severe Acute Respiratory Syndrome Coronavirus II, SARS-CoV-2 or COVID-19, is infecting and killing hundreds of African citizens, the impact of cholera, neglected due to the pandemic, is still rising very significantly. Therefore, the African continent is carrying a "double burden". Effectively, since 1817 and until now, cholera remains a global challenge to public health and an indicator of inequity and lack of social development [1].

Cholera affects approximately 2.9 million people yearly, leading to 95,000 deaths worldwide mainly in low- and middle-income countries. In Africa alone, 40 million people live in cholera-endemic areas with a risk of frequent outbreaks $[2,3]$. Throughout the centuries, African countries have been battling many outbreaks. However, the damage induced by the COVID-19 pandemic exceeds

\footnotetext{
*Correspondence: uwolivier1@ktu.edu.tr

1 Oli Health Magazine Organization, Research and Education, Kigali, Rwanda

Full list of author information is available at the end of the article
}

all previous and modern disease outbreaks in terms of the scope, extent, and persistence of its effects, which could undo decades of gains in public health and poverty reduction across the region [4].

Severe concerns have been pointed out regarding the flare-up of cholera during the COVID-19 pandemic. There has been an increase in reported cholera cases in endemic areas compared to previous years. The COVID-19 pandemic has disturbed the diagnosis and treatment services, and vaccination campaigns, including cholera vaccines, therefore, putting millions of lives at risk of vaccine-preventable disease $[5,6]$. Furthermore, the COVID-19 pandemic has caused a lack of access to humanitarian aid and also an increased pressure on health systems in cholera-endemic countries, making it impossible to handle two or more simultaneous outbreaks due to lack of capacity in personnel and supplies [7]. Additionally, the World Health Organization (WHO) and United Nations Children's Fund (UNICEF) have experienced challenges in dealing with the recent cholera outbreaks in many African Countries amidst the COVID-19 pandemic as it has 
added an extra burden on the already existing, limited resources $[8,9]$.

However, the pandemic has helped in spreading awareness on essential infection control measures: it has highlighted the importance of personal hygiene practices, the importance of vaccines in the protection against infectious disease and the role of prediction, preparedness, and early response that should be deployed to slow the spread of cholera [2].

With the COVID-19 outbreak, the increased threat of cholera should not be overlooked, especially that all regional health organizations are weighed down by this new pandemic. At this stage, international and humanitarian bodies may be essential to equip and prepare the African institutions to face and minimize the impact of even any potential pandemic in the future.

\section{The situation of fighting cholera before COVID 19 pandemic in Africa}

In March 2020, WHO declared COVID 19 arising from Wuhan province in China as a global pandemic and as a global threat [10]. This devastating news affected almost every sector including shifting attention from the existing fatal diseases that have claimed many people's lives far more than the COVID 19 [11].

Cholera has caused havoc for centuries affecting almost every continent, currently with a higher incidence in developing countries, being associated with poverty [12]. As cholera remains a significant global public health concern, its occurrence is linked to insufficient access to safe water and proper sanitation. Oral cholera vaccine $(\mathrm{OCV})$ has been recommended as an additional public health tool along with the safe Water, Sanitation and Hygiene (WASH) technique as a measure to eliminate the disease [13]. Before the emergence of vaccine, the WASH strategy was widely adopted in the fight against the disease in African countries [13].

OCV seems to be cost-effective as part of the prevention and control measure of cholera when targeted at the population with a high risk of cholera and poor access to healthcare facilities and has been employed in some countries of Africa, such as Malawi, Mozambique, and Ethiopia [14].

In 2011, WHO established a global oral cholera vaccine stockpile aiming at the rapid response to cholera outbreaks. From 2013 to 2017, over 25 million doses have been requested from the cholera vaccine stockpile, but only $51 \%$ were shipped to countries in need some African countries such as Malawi and Mozambique $[14,15]$.

\section{Burden of cholera during the COVID 19 outbreak in Africa}

Cholera outbreak remains a major public health concern since its first outbreak $[2,3,16]$. High-risk areas are periurban slums, concentration camps for displaced populations or refugees where the minimum requirement for clean and safe water and sanitation system requirements is not always met [1]. Disruption of humanitarian services to high-risk areas poses great setbacks in the fight against the cholera outbreak due to poor surveillance of the outbreak.

Due to lack of effective surveillance and weakening of healthcare system as efforts are being directed towards fight against COVID-19, data regarding cholera outbreaks are limited and uncertain in most African countries [1]. However, recently in the past 2 years, cholera outbreaks have been reported in Ethiopia and Sudan, both cholera-endemic regions coinciding with COVID19 era [17]. This has been evidenced in reports from Ethiopia, where disruption of response to cholera response during COVID-19 has led to approximately 15,000 cases and 250 deaths [17]. Conflicts also contributed to poor response to cholera outbreak, especially in the Tigray region, which caused Ethiopians to flee to Sudan, staying in refugee camps with disrupted WASH services. Disruption of WASH services coinciding with restrictions of humanitarian services, and the economic strains developed during COVID-19 are a great hindrance to campaigns against cholera outbreak, thus worsening the burden.

The last cholera outbreak in Somalia dates back to 2017 following a flood that occurred in Jubba basin and Shabelle River. The outbreak was contained in five out of six regions. However, as of April 2020, an increased number of cases was reported following a flood caused by heavy rainfalls, with the cumulative attack rate increasing to 26 persons per 100,000 by 2021 [8]. These findings suggest the role of naturally occurring events such as a flood in spreading the outbreak, but on the other hand, this presents critical failure of healthcare system response to cholera outbreak which occurred mainly during COVID19 era. In addition to cholera, some African countries have faced other infectious diseases and viral outbreaks such as bird flu, malaria, Ebola, measles, dengue, plague and Lassa fever [18-25].

\section{Efforts and challenges to cholera response amidst COVID-19 in Africa}

The widespread cholera epidemics in Africa have been linked to overpopulation, water, sanitation issues, and penury [26]. The prevention of cholera is focused on improving sanitation and hygiene standards. The 


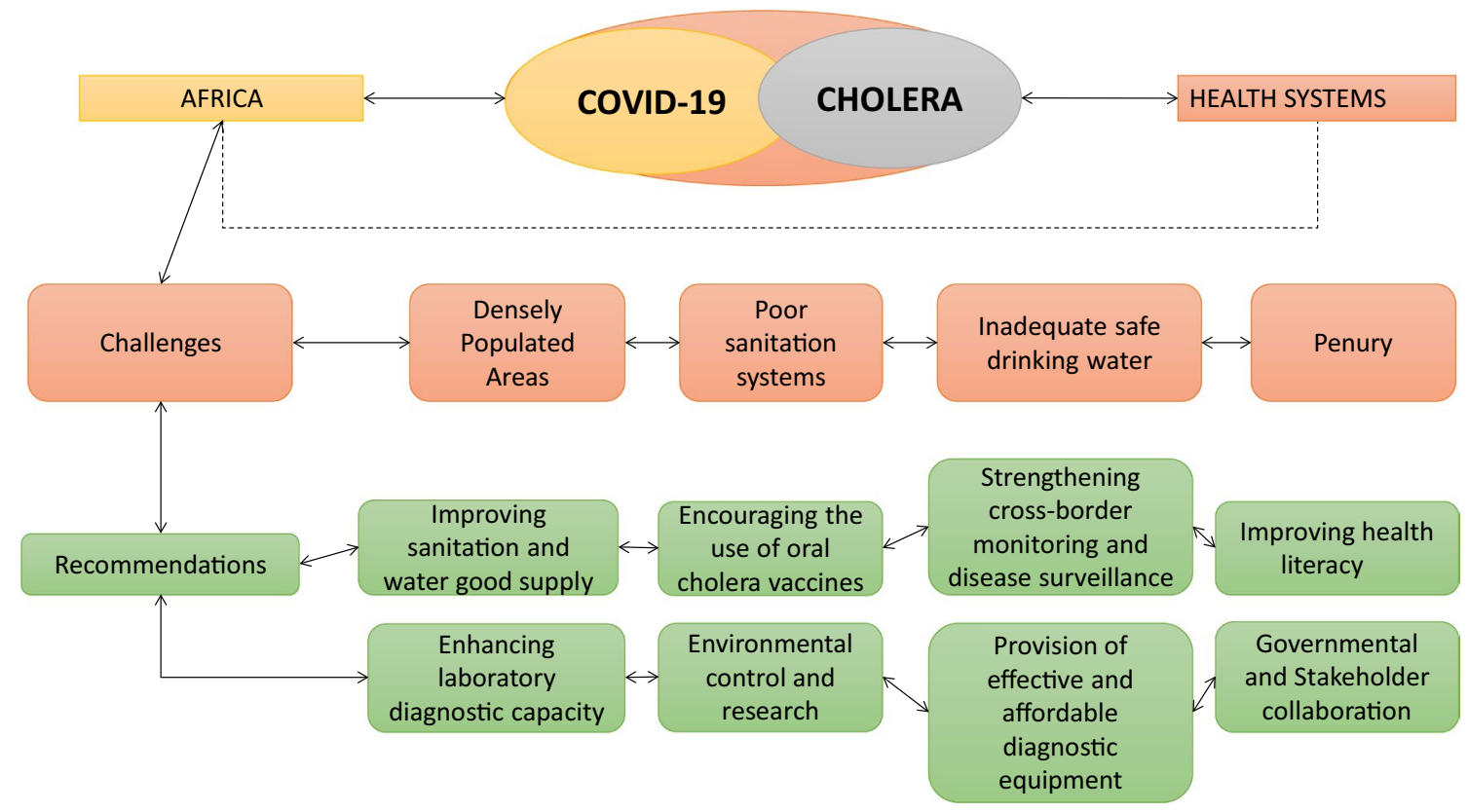

Fig. 1 A summary of challenges, priorities, and recommendations for the restriction of cholera pathogen amid the COVID-19 pandemic

World Health Organization (WHO) also endorses the oral cholera vaccines usage as a temporary preventative measure. There are several hurdles such as fragile socio-economic infrastructure, lack of adequate resources, logistics impeding widespread and pragmatic use of cholera vaccines in Africa, and vaccination rates remain low [27]. Cholera prevention and control strategy was adopted by 47 African countries prior to the COVID-19 pandemic. The African countries have agreed to reduce cholera incidence by implementing evidence-based steps, strengthening treatment methods, strengthening border monitoring, and encouraging oral cholera vaccines use while expanding water supply investments [28]. The pandemic, as expected, had an adverse influence on many schemes that could be implemented, such as vaccine advertising efforts, supplies, launching surveillance system, hygiene programs that were victimized due to lockdown initiatives, quarantine and social distance to help deter spreading the disease. Therefore, national control and prevention strategies should be implemented, providing effective and affordable diagnostics and treatment for cholera and COVID-19, enhancing laboratory diagnostics, clinical management, environmental control and research. The community's involvement is highly recommended, and the development of an effective prevention strategy would be the key pillars to mitigate cholera incidence, especially during the challenging times of the COVID19 pandemic.

\section{Recommendations}

The emergence of COVID-19 has disrupted the hard-won years of progress against cholera in Africa, but despite the paradigm shift in focus on COVID-19, it is critical that cholera must not become more forgotten. While the focus is on COVID-19, efforts such as improving water supply investment and sanitation, encouraging the use of oral cholera vaccines, strengthening cross-border surveillance, government, and stakeholder collaboration are necessary to counter the burden of cholera. A summary of challenges and recommendations is provided in the figure below (Fig. 1).

\section{Conclusion}

Africa is a high-risk area for the spread of cholera due to poor sanitation systems, lack of good drinking water, and densely populated areas. Disruption of healthcare services in high-risk areas due to COVID-19 therefore poses a great challenge in the fight against cholera. As priority is now being shifted towards COVID-19 containment, data regarding cholera outbreaks are limited and unavailable in most African countries resulting in a lapse in contact tracing and the management of cholera patients as they are left undiagnosed and untreated. To win the fight against cholera and COVID-19 simultaneously, a 'whole of Africa' integrated approach involving collective advocacies, cross-border surveillance and information sharing, and stakeholder collaboration would be crucial for 
progress. This will ensure that no country is left behind because no one is safe until everyone is safe.

\section{Abbreviations}

COVID-19: Coronavirus Disease 2019; WHO: World Health Organization; UNICEF: United Nations Children's Emergency Fund; OCV: Oral cholera vaccine; WASH: Water, Sanitation and Hygiene.

\section{Acknowledgements}

None.

\section{Authors' contributions}

OU: conceptualization, project administration, writing — review and editing, and designing. HO: reviewed and edited the first and second draft. Collection and assembly of data: all authors. Data analysis and interpretation: all authors. Manuscript writing: all authors. All authors read and approved the final manuscript.

\section{Funding}

We have not received any financial support for this manuscript.

\section{Availability of data and materials}

Not applicable.

\section{Declarations}

\section{Ethics approva}

Not applicable.

\section{Competing interests}

The authors declared no competing interests.

\section{Author details}

${ }^{1}$ Oli Health Magazine Organization, Research and Education, Kigali, Rwanda. ${ }^{2}$ Clinton Global Initiative University, New York, USA. ${ }^{3}$ Faculty of Medicine, Karadeniz Technical University, 61080 Trabzon, Turkey. ${ }^{4}$ Faculty of Pharmaceutical Sciences, University of Ilorin, Kwara State, Nigeria. ${ }^{5}$ School of Chemical Engineering, University of Birmingham, Edgbaston, Birmingham B15 2TT, UK. ${ }^{6}$ Department of Biochemistry and Molecular Biology, Faculty of Life Science, Mawlana Bhashani Science and Technology University, Tangail 1902, Bangladesh. ${ }^{7}$ University of Dar es salaam Tanzania, Dar es Salaam, Tanzania. ${ }^{8}$ Mbeya Zonal Consultant Hospital, Mbeya, Tanzania. ${ }^{9}$ Department of Surgery, University of Ilorin Teaching Hospital, Kwara State, Nigeria. ${ }^{10}$ Faculty of Medicine, University of Saint Joseph Beirut, Beirut, Lebanon. ${ }^{11}$ Department of Obstetrics and Gynaecology, Olabisi Onabanjo University Teaching Hospital, Sagamu, Nigeria. ${ }^{12}$ Faculty of Health Sciences, McMaster University, Hamilton, ON, Canada.

Received: 21 July 2021 Accepted: 1 October 2021

Published online: 24 November 2021

\section{References}

1. Hassan OB, Nellums LB. Cholera during COVID-19: the forgotten threat for forcibly displaced populations. EClinicalMedicine. 2021;32: 100753. https://doi.org/10.1016/j.eclinm.2021.100753.

2. COVID-19, infection control, and cholera, 2021. https://www.healtheuro pa.eu/covid-19-infection-control-and-cholera/107561/. Accessed 10 May 2021

3. Ali M, Lopez AL, You YA, Kim YE, Sah B, Maskery B, Clemens J. The global burden of cholera. Bull World Health Organ. 2012;90:209-18. https://doi. org/10.2471/BLT.11.093427.

4. Disease Surveillance, Emergency Preparedness and Response in Eastern and Southern Africa-World | ReliefWeb, 2021. https://reliefweb.int/ report/world/disease-surveillance-emergency-preparedness-and-respo nse-eastern-and-southern-africa. Accessed 10 May 2021.
5. COVID-19: massive impact on lower-income countries threatens more disease outbreaks | Gavi, the Vaccine Alliance, 2021. https://www.gavi. org/news/media-room/covid-19-massive-impact-lower-income-count ries-threatens-more-disease-outbreaks. Accessed 10 May 2021.

6. Impact of COVID-19 on vaccine supplies / UNICEF Supply Division, 2020 https://www.unicef.org/supply/stories/impact-covid-19-vaccine-suppl ies. Accessed 10 May 2021.

7. Deadly malaria and cholera outbreaks grow amongst refugees as COVID pandemic strains health systems, warns IRC | International Rescue Committee (IRC), 2020. https://www.rescue.org/press-release/deadly-malar ia-and-cholera-outbreaks-grow-amongst-refugees-covid-pandemic-strai ns. Accessed 10 May 2021.

8. WHO EMRO | Outbreak update-Cholera in Somalia, 24 January 2021 | Cholera | Epidemic and pandemic diseases, 2021. http://www.emro.who. int/pandemic-epidemic-diseases/cholera/outbreak-update-cholera-insomalia-24-january-2021.html. Accessed 10 May 2021.

9. Advocacy brief: a water crisis in the time of COVID-19: impact of funding shortages on Public Health in Yemen, June, 2020-Yemen | ReliefWeb, 2020. https://reliefweb.int/report/yemen/advocacy-brief-water-crisistime-covid-19-impact-funding-shortages-public-health-yemen. Accessed 10 May 2021.

10. Shereen MA, Khan S, Kazmi A, Bashir N, Siddique R. COVID-19 infection: origin, transmission, and characteristics of human coronaviruses. J Adv Res. 2020;24:91-8. https://doi.org/10.1016/j.jare.2020.03.005.

11. Paul E, Brown GW, Ridde V. COVID-19: time for paradigm shift in the nexus between local, national and global health. BMJ Glob Health. 2020;5:2622. https://doi.org/10.1136/bmjgh-2020-002622.

12. Gabutti G, Rossanese A, Tomasi A, Giuffrida S, Nicosia V, Barriga J, Florescu C, Sandri F, Stefanati A. Cholera, the current status of cholera vaccines and recommendations for travellers. Vaccines. 2020;8:1-17. https://doi.org/10. 3390/vaccines8040606.

13. D'Mello-Guyett L, Gallandat K, Van Den Bergh R, Taylor D, Bulit G, Legros D, Maes P, Checchi F, Cumming O. Prevention and control of cholera with household and community water, sanitation and hygiene (WASH) interventions: a scoping review of current international guidelines. PLOS ONE. 2020. https://doi.org/10.1371/journal.pone.0226549.

14. Teoh SL, Kotirum S, Hutubessy RCW, Chaiyakunapruk N. Global economic evaluation of oral cholera vaccine: a systematic review. Hum Vaccines Immunother. 2018;14:420-9. https://doi.org/10.1080/21645515.2017. 1392422.

15. Hsiao A, Desai SN, Mogasale V, Excler JL, Digilio L. Lessons learnt from 12 oral cholera vaccine campaigns in resource-poor settings. Bull World Health Organ. 2017;95:303-12. https://doi.org/10.2471/BLT.16.175166.

16. WHO. Cholera, 2021. https://www.who.int/news-room/fact-sheets/detail/ cholera. Accessed 6 May 2021.

17. The Global Alliance Against Cholera (G.A.A.C). Ongoing cholera epidemic in Ethiopia, 2020. https://www.choleraalliance.org/en/ressources/news/ ongoing-cholera-epidemic-ethiopia. Accessed 6 May 2021

18. Uwishema O, Adriano LF, Chalhoub E, et al. Bird flu outbreak amidst COVID-19 pandemic in South Africa: efforts and challenges at hand. J Med Virol. 2021. https://doi.org/10.1002/jmv.27124.

19. Aborode AT, David KB, Uwishema O, Nathaniel AL, Imisioluwa JO, Onigbinde SB, Faroog F. Fighting COVID-19 at the expense of malaria in Africa: the consequences and policy options. Am J Trop Med Hyg. 2021;104(1):26-9. https://doi.org/10.4269/ajtmh.20-1181.

20. Aborode AT, Tsagkaris C, Jain S, Ahmad S, Essar MY, Fajemisin EA, Adanur 1. Uwishema O. Ebola outbreak amid COVID-19 in the Republic of Guinea: priorities for achieving control. Am J Trop Med Hyg. 2021. https://doi.org/ 10.4269/ajtmh.21-0228.

21. Uwishema O, Adriano LF, Torbati T, Onyeaka H. Measles crisis in Africa amidst the COVID-19 pandemic: delayed measles vaccine administration may cause a measles outbreak in Africa. J Med Virol. 2021. https://doi.org/ 10.1002/jmv.27150

22. Uwishema O, Adanur I, Babatunde AO, Hasan MM, Elmahi O, Olajumoke KB, Aborode AT, Emmanuella N, Costa A, Ahmad S, Essar MY. Viral infections amidst COVID-19 in Africa: implications and recommendations. J Med Virol. 2021. https://doi.org/10.1002/jmv.27211.

23. Uwishem $\mathrm{O}$, et al. Dengue fever outbreak in Cook Island: a rising concern, efforts, challenges and future recommendations. J Med Virol. 2021. https://doi.org/10.1002/jmv.27223. 
24. Aborode AT, Dos Santos Costa AC, Mohan A, Goyal S, Rabiu AT, Tsagkaris C, Uwishema O, Outani O, Ahmad S, Essar MY. Epidemic of plague amidst COVID-19 in Madagascar: efforts, challenges, and recommendations. Trop Med Health. 2021;49(1):56. https://doi.org/10.1186/s41182-021-00349-5.

25. Uwishema O, et al. Lassa fever amidst the COVID-19 Pandemic in Africa: a rising concern, efforts, challenges and future recommendations. J Med Virol. 2021. https://doi.org/10.1002/jmv.27219.

26. Cholera $|\mathrm{WHO}|$ Regional Office for Africa, 2021. https://www.afro.who. int/health-topics/cholera. Accessed 6 May 2021.

27. WHO EMRO |Cholera | Health topics, 2021. http://www.emro.who.int/ health-topics/cholera/index.html. Accessed 6 May 2021.
28. African Health Ministers commit to ending cholera outbreaks by 2030 | WHO | Regional Office for Africa, 2021. https://www.afro.who.int/news/ african-health-ministers-commit-ending-cholera-outbreaks-2030. Accessed 6 May 2021.

\section{Publisher's Note}

Springer Nature remains neutral with regard to jurisdictional claims in published maps and institutional affiliations.
Ready to submit your research? Choose BMC and benefit from:

- fast, convenient online submission

- thorough peer review by experienced researchers in your field

- rapid publication on acceptance

- support for research data, including large and complex data types

- gold Open Access which fosters wider collaboration and increased citations

- maximum visibility for your research: over $100 \mathrm{M}$ website views per year

At BMC, research is always in progress.

Learn more biomedcentral.com/submissions 